\title{
Suicidal Ideation, CTCAE
}

National Cancer Institute

\section{Source}

National Cancer Institute. Suicidal Ideation, CT CAE. NCI Thesaurus. Code C143864.

A disorder characterized by thoughts of taking one's own life. 